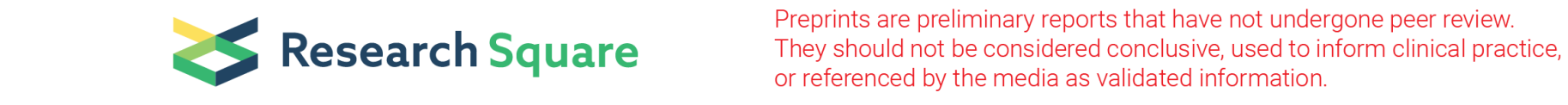

\title{
Clinical Features and Treatment Outcomes of Vulvar Sarcoma: A Single- Institution Review of 21 Cases
}

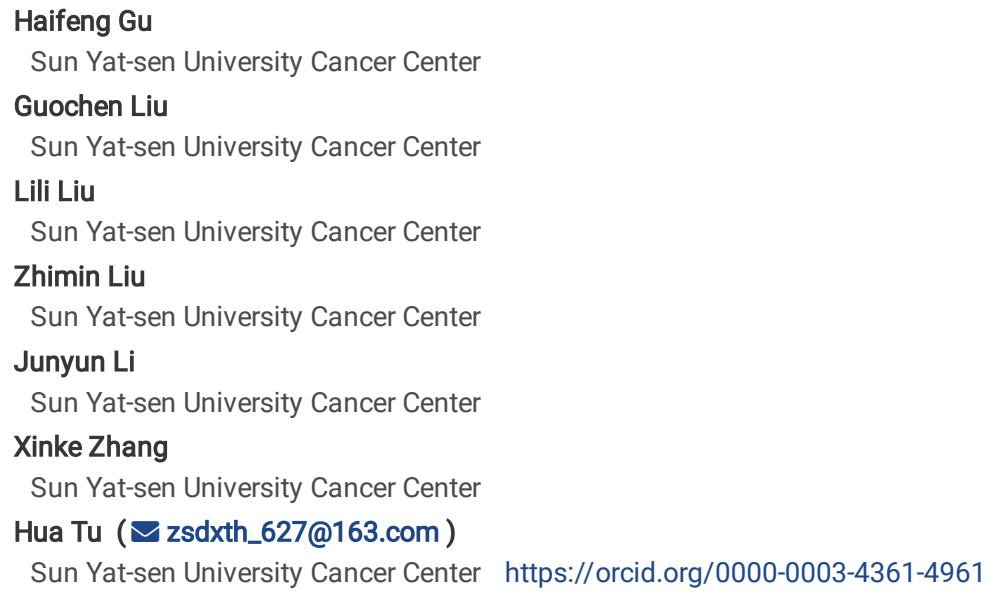

Sun Yat-sen University Cancer Center

Guochen Liu

Sun Yat-sen University Cancer Center

Lili Liu

Sun Yat-sen University Cancer Center

Zhimin Liu

Sun Yat-sen University Cancer Center

Junyun Li

Sun Yat-sen University Cancer Center

Xinke Zhang

Sun Yat-sen University Cancer Center

Hua Tu ( $\square$ zsdxth_627@163.com)

Sun Yat-sen University Cancer Center https://orcid.org/0000-0003-4361-4961

Research

Keywords: Vulvar sarcoma, surgery, pathology, recurrence, prognosis

Posted Date: June 9th, 2020

DOI: https://doi.org/10.21203/rs.3.rs-34048/v1

License: (c) (i) This work is licensed under a Creative Commons Attribution 4.0 International License. Read Full License 


\section{Abstract}

\section{Background}

Valvar sarcoma is an extremely rare disease in which the data for treatment and prognosis are scarce. The aim of this study was to analysis the clinical features and treatment outcomes of initial and recurrent valvar sarcoma.

\section{Method}

The medical records of patients with initial or recurrent vulvar sarcoma treated at Sun Yat-sen University Cancer Center between January 2005 and April 2019 were retrospectively reviewed.

\section{Results}

A total of 21 patients with vulvar sarcoma were identified. The median age of them was 43 years (range: 16 to 71 ). The histological subtypes were epithelioid sarcoma, leiomyosarcoma, dermatofibrosarcoma protuberans, rhabdomyosarcoma, and angiosarcoma in 9, 4, 4, 3 and 1 patients, respectively. None of the patients received wide resection when they were initially treated at communities. Thirteen patients were referred immediately after initial managements (Group A), whereas 8 patients were referred with recurrent disease (Group B). Wide resections were performed with pathologically negative margins in 20 patients, of whom 9 also received lymphadenectomy and 3 showed metastases. One patient in Group B only received chemoradiotherapy for unresectable pelvic mass. Adjuvant chemoradiotherapy were performed for 12 patients evaluated to have high risk of recurrence. The median follow-up time was 39 months (range: 11 to 174) for Group A and 107 (range: 2 to 139) for Group B. During the follow-up, relapse occurred in 3 patients in Group A and 5 in Group B. The 3- and 5-year survival rates were $92.3 \%$ and $76.9 \%$ for Group A, $50.0 \%$ and $37.5 \%$ for Group B, respectively.

\section{Conclusions}

VS is usually resected as benign disease at initial management. Immediate extended resection with negative margins provided favorable outcomes for most of the patients. The prognosis for recurrent cases were poor, however there remained survival opportunities if aggressive treatments were carried out.

\section{Introduction}

Vulvar sarcoma (VS) is an extremely rare disease that accounts for 1 3\% of all vulvar cancers [1]. It is an aggressive malignancy that usually affects women of reproductive age [2]. The histological type of VS is heterogeneous and mainly includes leiomyosarcoma, dermatofibrosarcoma protuberans, angiosarcoma, rhabdomyosarcoma and epithelioid sarcoma $[3,4]$. Unlike other vulvar malignancies, VS usually grows with benign appearance and non-specific manifestation, which increases the difficulty of early diagnosis [5]. The biological behavior of VS is featured by frequent recurrence and high potential to metastasize, for which the initial treatment is quite important [6].

Most available data on VS are case reports and no practical guideline has been established [7, 8]. The data on recurrent VS is even fewer. According to the National Comprehensive Cancer Network guidelines for soft tissue sarcomas, the treatment should be based on surgical resection with wide margin [9]. Due to the anatomic particularity of VS, wide resection usually requires a reconstructive procedure, making the surgery more challenging. On the other hand, the role of lymphadenectomy remains unclear since there are few data on this aspect [10,11]. In a review of 28 case reports of vulvar epithelioid sarcoma, lymph node metastasis occurred in $46.2 \%(6 / 13)$ recurrent cases, whereas none of them received lymphadenectomy at initial surgery [12]. Thus, it is necessary to evaluate the therapeutic value of lymphadenectomy for both initial and recurrent VS.

Due to the rarity of VS, no survival data has been summarized in a single study. Besides, the characteristics and prognosis of recurrent VS are largely unknown. Here we present a report of 21 patients with VS treated at Sun Yat-sen University Cancer Center. Many of them had a history of recurrence. The aim of this study was to analysis the clinical features and treatment outcomes of VS and provide new evidence for clinical practice of this rare disease.

\section{Methods}

The medical records of patients with vulvar cancer treated at Sun Yat-sen University Cancer Center between January 2005 and April 2020 were reviewed with Institutional Review Board approval. Twenty-one patients with histologically confirmed VS were identified from 543 patients with vulvar cancer. All of them were Chinese and the median age was 43 years (range: 16 to 71). The histological subtypes included 9 epithelioid sarcomas, 4 leiomyosarcomas, 4 dermatofibrosarcoma protuberans, 3 rhabdomyosarcomas, and 1 angiosarcoma. All pathological sections were re-reviewed by a pathologist to confirm the diagnoses. Immunohistochemical (IHC) examination was applied for all initial specimens and Fluorescence in Situ Hybridization (FISH) examination was performed in 6 patients. Informed consent to treatment were obtained for all patients.

Individualized surgical strategy was applied, in which the boundaries of resection were tailored with enough margins to the tumor or prior incision. The width of margins relied on evaluation of the tumor size and location, risk of relapse, and the difficulty of suture. The depth of resection reached the urogenital diaphragm. Pedicled flap transplantation was performed when it was considered necessary and feasible. Lymphadenectomy was selectively performed according to preoperative evaluation. Postoperative treatments included radiotherapy and chemotherapy, which were determined with multidisciplinary consultation. All radiation fields were delineated by senior radiologists. The chemotherapy regimens were formulated individually.

The clinical-pathological data and treatment outcomes were obtained from hospital information system, institutional follow-up department, and when necessary through contact with the patient's family. Survival time was calculated from the first hospitalization in our center to the cancer-related death or last 
follow-up. Survival curves were plotted using the Kaplan-Meier method and compared by the log-rank test. Statistical analysis was performed using Statistical Product and Service Solutions software (version 19.0, SPSS Inc., Chicago, IL, USA) with a p-value of $<0.05$ considered statistically significant.

\section{Results}

The data of 21 patients with VS are listed in Table 1. Majority of them were presented with painless subdermal mass. The median diameter of the primary tumor was $3 \mathrm{~cm}$ (range: 1.5 to 8). All of them were initially treated at communities and none of the disease was confirmed or suspected as VS before treatment. The initial management was simple resection in 20 patients and wedge-shaped biopsy in 1 (No.18). After initial pathological examination, 19 patients got the diagnosis of VS while 2 were misdiagnosed as other malignancies. Thirteen patients were referred to our institution immediately after initial managements (Group A), whereas 8 patients were referred with recurrent disease (Group B). None of them showed distant metastasis when referred. In Group $B$, the recurrences were the first, second and third time in 4, 2 and 2 patients, respectively. IHC examination was performed for all patients using panels of biological markers. The results of IHC examination for each patient are detailed in Supplementary Table 1.

There was no special concern in the previous histories of these patients except the NO. 6 patient. She suffered breast and endometrial cancer, respectively, 13 and 10 years before the occurrence of vulvar dermatofibrosarcoma protuberans, which was diagnosed with FISH examination showing positive signals for COL1A1-PDGFB fusion.

In Group A, patients were treated with extended local resection with 2 to $2.5 \mathrm{~cm}$ margins. Flap transplantation was performed in 2 patients. Two patients showed microscopic disease despite there was no apparent tumor left. Inguinal lymphadenectomy was performed in 5 patients due to palpable nodes or suspected imageology and the results were positive in 1 (No.2). The treatment modality was distinctive for No. 18 patient who received wedge-shaped biopsy at community due to massive tumor involving the anus. We planned an extensive resection including anal excision and colostomy. However, she refused this scheme and consented to receive neoadjuvant chemotherapy first. After 3 cycles of chemotherapy the tumor got partial remission. We performed a less extensive resection in which flap transplantation was performed and the function of anus was preserved. Although negative margins were achieved and adjuvant chemoradiotherapy was carried out, she died of disease 11 months after the operation.

Mild to moderate skin necrosis and wound infection occurred in 3 patients undergoing flap transplantation or inguinal lymphadenectomy. The median hospital stay was 10 days (range: 5 to 30 ). Patients with residual disease, lymph node metastasis, large tumor or mitoses $>20$ per 10 high power field were submitted to chemotherapy or radiotherapy. Radiotherapy were carried out with a median dosage of 46 Gy (range: 36 to 60 ) in 18 to 25 fractions. The agents for chemotherapy mainly consisted of ifosfamide, cisplatin, dacarbazine, epirubicin and liposomal doxorubicin.

In Group B, many patients had received more than one vulvar operation at other hospitals and were referred with massive tumors. None of them received wide resection at initial management. The goal of surgery was to remove the tumor with as wide margins as possible. Flap transplantation was performed in 4 patients, of whom one received flap transplantation again in the surgery for later relapse. Inguinal lymphadenectomy was performed in 4 patients, of whom 2 (No.8 and 16) also received retroperitoneal lymphadenectomy and showed metastasis. The surgery was abandoned and replaced by palliative chemoradiotherapy in No.12 patient because of unresectable pelvic mass.

After surgery, 6 patients suffered moderate to severe complications including skin necrosis, lymphatic fistula, lymphedema, and wound infection. The median hospital stay was 22 days (range: 2 to 39). Adjuvant chemotherapy and radiotherapy were planned in 7 patients. The policy of adjuvant treatment was similar to those for Group A.

The median follow-up time calculated by reverse Kaplan-Meier method was 39 months (range: 11 to 174) for Group A and 107 (range: 2 to 139 ) for Group B. During the follow-up, relapse occurred in 3 patients in Group A and 5 in Group B. All of them received secondary surgery followed by chemotherapy or radiotherapy and only No.1 patient in Group A still survived with metastasis at last follow-up. Distant metastasis occurred in 5 patients, with the sites including the liver, lung, lumbar and brain. The 3 - and 5-year survival rates were $92.3 \%$ and $76.9 \%$ for Group A, 50.0\% and $37.5 \%$ for Group B, respectively. The difference in survival between the two groups approached significance $(P=0.078)$. The survival curves are displayed in Figure 1.

\section{Discussion}

In this study, we summarized the data of 21 Chinese patients with VS treated at Sun Yat-sen University Cancer Center. To our knowledge, this is the largest cohort to date from single institution, allowing for the calculation of survival rates. The 3-year survival rate was $92.3 \%$ for patients receiving wide resection at initial diagnosis, and $50.0 \%$ for those referred with recurrent disease. Although the difference merely approached statistical significance, it clearly indicated the importance of wide resection in the initial management of VS.

VS is a heterogeneous disease with several histological subtypes $[3,4]$. In our institution, VS accounted for $3.9 \%$ of contemporaneous vulvar malignancies. Epithelioid sarcoma was the most common subtype in our cohort (42.9\%), followed by leiomyosarcoma (19.0\%) and dermatofibrosarcoma protuberans (19.0\%). The onset age covered a wide range in patients with epithelioid sarcoma and dermatofibrosarcoma protuberans, while it seemed to be younger for patients with leiomyosarcoma and rhabdomyosarcoma. Due to the diversity of its histological subtypes, pathologists usually encounter difficulty in diagnosis, necessitating additional diagnostic measures $[13,14]$. In our study, all patients had IHC examination with panels of multiple biological markers. The expression profile for each histological subtype was generally the same to those reported in previous literatures.

FISH was performed in 6 patients and only one patient with dermatofibrosarcoma protuberans showed positive signals for COL1A1-PDGFB fusion. Dermatofibrosarcoma protuberans is a relatively less aggressive tumor featured by high incidence of local recurrence and low risk of metastases [15]. A recent case report by Neff et al. described a case with vulvar massive dermatofibrosarcoma protuberans [16]. Although the tumor had invaded the deep inguinal-

Page $3 / 9$ 
femoral spaces, the surrounding lymph nodes were negative. However, in our cohort, No.2 patient displayed a different disease course, in which multiple inguinal node metastases occurred despite relatively small tumor $(3 \times 4 \mathrm{~cm})$. The pathological graphics of this patient are showed in Figure 2 . This patient finally died of the disease although extensive resection and adjuvant chemoradiotherapy were accomplished, suggesting the importance of evaluation on regional nodes and the necessity of additional therapeutic approaches.

COL1A1-PDGFB gene rearrangements can be detected in majority of dermatofibrosarcoma protuberans [17]. Jahanseir et al. reported a series of 11 patients with vulvar dermatofibrosarcoma protuberans, of whom 9 were found to have COL1A1-PDGFB gene rearrangement [18]. The COL1A1-PDGFB fusion produces a chimeric protein, which can stimulate cell proliferation through maintaining the activity of platelet-derived growth factor receptor [19]. This molecular mechanism relies on the function of downstream tyrosine kinases which can be utilized as therapeutic target [20]. Notedly in our study, the patient showing positive for COL1A1-PDGFB fusion also had a history of breast and endometrial cancers, suggesting an important role of COL1A1-PDGFB fusion in the course of tumorigenesis. Thus, for patients with suspected vulvar dermatofibrosarcoma protuberans, FISH should be recommended not only for diagnostic purposes but also for potential targeted therapy.

Radical resection with enough margin is a guarantee of oncological control for VS [6,21]. Unfortunately, all patients in our cohort were initially treated at community and none of them received wide resection at primary surgery. For patients who were timely referred to our gynecological oncologists, extended resection resulted in favorable outcomes at minor costs. Most of these patients were discharged within one week after operation and there were no severe complications observed. Only 3 patients relapsed during a median follow-up period of 36 months.

The No.1 patient is a special case who was initially misdiagnosed as Bartholin cyst and treated by cystectomy. She was referred to us with revised pathological diagnosis of well-differentiated leiomyosarcoma and then received extended resection with negative margins. Four years later she got relapse at vulva and received the third resection. Again, four years later she got the second relapse simultaneously at vulva and lumbar vertebra. Her disease was evaluated as progress after 3 cycles of chemotherapy (ifosfamide+cisplatin+doxorubicin). Afterwards, she sequentially received the fourth vulvar surgery, radiotherapy to the lumbar, and immunotherapy using cytokine induced killer cells. She had survived with disease more than 5 years after the last treatment and the disease was evaluated as stable in a most recent follow-up (March 2020). The treatment course of this patient implied high relapse potential of leiomyosarcoma and underlined the importance of initial surgery. Furthermore, multidisciplinary cooperation might be a solution for metastatic diseases.

There are very few data on recurrent VS in the literature. In our cohort, 8 patients were referred with at least one recurrence, for whom the treatment became more challenging. The surgeries for them were more extensive in which adjacent structure resection and flap transplantation were usually required. In addition, chemoradiotherapy was also planned in 7 patients. The surgical morbidity was high, and the hospital stays were obviously longer than those referred at initial diagnosis. Nevertheless, the outcomes seemed to be encouraging since there were 3 patients achieved long-term survival without disease (No.3, 7, 14). Therefore, we believe that the recurrent VS remains potentially curable and aggressive treatments should be planned especially for patients with local recurrence.

The role of lymphadenectomy in the treatment for VS is unclear [12]. Theoretically, sarcomas spread mainly by hematogenous pathway and lymphadenectomy can be omitted unless there are clinically suspected nodes [22]. However, the rate of nodal metastasis seemed to be higher in some subtypes of sarcoma, such as epithelioid sarcoma and rhabdomyosarcoma [23]. In our cohort 9 patients had lymphadenectomy and 3 of them had positive findings. However, we could not confirm a therapeutic benefit of lymphadenectomy as all patients showing lymph node metastases had relapsed in a short time and finally died of disease. The necessity of lymphadenectomy may be determined by histological subtype and imaging examination. Given the high morbidity associated with lymphadenectomy, sentinel lymph node biopsy may serve as an alternative when indicated [24].

The role of chemoradiation as adjuvant treatment for VS is also unclear. Postoperative radiotherapy had been found to be beneficial for a variety of sarcomas [25]. Evidence from sarcoma of extremities suggested an improvement of recurrence-free survival in patients receiving postoperative chemotherapy [26]. The experience from our cohort showed that these adjuvant therapies might be beneficial in specific situations.

As a retrospective study of rare disease, our analysis was limited by its small sample size. There might also be unaware heterogeneity between different histological subtypes. Besides, nearly half of our patients were recurrent cases and the adjuvant treatments were highly individualized, which weakened the capacity to analyze the prognostic impact of single intervention. Thus, the results of this study should be interpreted with prudence.

In conclusion, VS is a rare malignance with heterogeneous histological types. IHC and FISH are important diagnostic measures. Although most patients were initially treated with inadequate surgery, additional extended resection by gynecological oncologists provided favorable outcomes. For recurrent cases, there remained some opportunities to survive if aggressive treatments were carried out. The therapeutic benefits of lymphadenectomy and chemoradiotherapy remains to be determined in further investigations.

\section{Declarations}

\section{Ethics approval and consent to participate}

This study was approved by the Institutional Review Board of Sun Yat-sen University Cancer Center.

\section{Consent for publication}

Not applicable.

\section{Availability of data and material}


The raw data of this paper are available upon reasonable request to the corresponding author.

\section{Competing interests}

The authors declare that they have no competing interests.

\section{Funding}

None.

\section{Authors' contributions}

HT contributed to the study conception and participated in the literature retrieval and data analyses. HG, GL, ZL and JL participated in the data collection. LL and XZ performed the pathological review and collected pathological data. HF-G, GC-L and HT drafted the manuscript. All authors read and approved the final manuscript.

\section{Acknowledgements}

Not applicable.

\section{Abbreviations}

VS: vulvar sarcoma; IHC, immunohistochemical; FISH, fluorescence in situ hybridization.

\section{References}

1. Redroban L, Montalvo N. Vulvar Myxoid Liposarcoma, an Extremely Rare Diagnosis: A Case Report and Review of Literature. Int J Gynecol Pathol 2019; 38(1): 17-20.

2. Han CH, Li X, Khanna N. Epithelioid sarcoma of the vulva and its clinical implication: A case report and review of the literature. Gynecol Oncol Rep 2016; 15: 31-33.

3. Chokoeva AA, Tchernev G, Cardoso JC, Patterson JW, Dechev I, Valkanov S et al. Vulvar sarcomas: Short guideline for histopathological recognition and clinical management. Part 1. Int J Immunopathol Pharmacol 2015; 28(2): 168-177.

4. Chokoeva AA, Tchernev G, Cardoso JC, Patterson JW, Dechev I, Valkanov S et al. Vulvar sarcomas: Short guideline for histopathological recognition and clinical management. Part 2. Int J Immunopathol Pharmacol 2015; 28(2): 178-186.

5. Rodrigues Al, Lopes HI, Lima O, Marta S. Proximal-type epithelioid sarcoma-unusual presentation: unilateral vulvar mass. BMJ Case Rep 2015 ; 2015.

6. Khazeni K, LaBove H, Wilky B, Rosenberg AE, Paulus E, Chim H et al. Myoepithelial carcinoma or epithelioid sarcoma - A rare diagnosis with poor prognosis. A case report and review of literature. Int J Surg Case Rep 2018; 49: 239-243.

7. Sundaram A, Elangovan A, Rajwanshi A, Srinivasan R, Kapoor R. Proximal-type epithelioid sarcoma of the vulva: Cytopathological diagnosis of a rare neoplasm. Cytopathology 2018; 29(5): 471-473.

8. Schoolmeester JK, Leifer AJ, Wang L, Hameed MR. Vulvar Myxoid Liposarcoma and Well Differentiated Liposarcoma With Molecular Cytogenetic Confirmation: Case Reports With Review of Malignant Lipomatous Tumors of the Vulva. Int J Gynecol Pathol 2015; 34(4): 390-395.

9. NCCN-National Comprehensive Cancer Network. NCCN Clinical Practice Guidelines in Oncology: Soft Tissue Sarcoma. Version 6.2019.

10. Kasamatsu T, Hasegawa T, Tsuda H, Okada S, Sawada M, Yamada T et al. Primary epithelioid sarcoma of the vulva. Int J Gynecol Cancer 2001; 11(4): 316-320.

11. Sun L, Wu LY, Li XG, Bai P, Zhang HT. Clinical characterization of vulvar epithelioid sarcoma. Zhonghua Zhong Liu Za Zhi 2010; 32 (12): $935-938$.

12. lavazzo C, Gkegkes ID, Vrachnis N. Dilemmas in the management of patients with vulval epithelioid sarcoma: a literature review. Eur J Obstet Gynecol Reprod Biol 2014; 176: 1-4.

13. De Leo A, Ricci C, Terzano P, Santini D, Corti B. Atypical fibroxanthoma associated with differentiated-type vulvar intraepithelial neoplasia: Case report of an unusual entity. JAAD Case Rep 2019; 5(5): 448-450.

14. Gilani S, Al-Khafaji B. Dermatofibrosarcoma protuberans of the vulva: a mesenchymal tumour with a broad differential diagnosis and review of literature. Pathologica 2014; 106(4): 338-341.

15. Vathiotis IA, Psichogiou E, Syrigos KN, Kotteas EA. Lung Metastasis From Fibrosarcomatous Dermatofibrosarcoma Protuberans of the Vulva: A Rare Case Report. J Low Genit Tract Dis 2018; 22(1): 85-87.

16. Neff R, Collins R, Backes F. Dermatofibrosarcoma protuberans: A rare and devastating tumor of the vulva. Gynecol Oncol Rep 2019; 28 : 9-11.

17. Li N, Zhou T, Chen S, Yang R, Zhu Q, Feng Z. COL1A1-PDGFB gene fusion in dermatofibrosarcoma protuberans: a useful diagnostic tool and clinicopathological analysis. Int J Clin Exp Pathol 2018; 11(8): 4052-4059.

18. Jahanseir K, Xing D, Greipp PT, Sukov WR, Keeney GL, Howitt BE et al. PDGFB Rearrangements in Dermatofibrosarcoma Protuberans of the Vulva: A Study of 11 Cases Including Myxoid and Fibrosarcomatous Variants. Int J Gynecol Pathol 2018; 37(6): 537-546.

19. Shimizu A, O'Brien KP, Sjoblom T, Pietras K, Buchdunger E, Collins VP et al. The dermatofibrosarcoma protuberans-associated collagen type lalpha1/platelet-derived growth factor (PDGF) B-chain fusion gene generates a transforming protein that is processed to functional PDGF-BB. Cancer Res 
1999; 59(15): 3719-3723.

20. Rubin BP, Schuetze SM, Eary JF, Norwood TH, Mirza S, Conrad EU et al. Molecular targeting of platelet-derived growth factor B by imatinib mesylate in a patient with metastatic dermatofibrosarcoma protuberans. J Clin Oncol 2002; 20(17): 3586-3591.

21. Kainhofer V, Smolle MA, Szkandera J, Liegl-Atzwanger B, Maurer-Ertl W, Gerger A et al. The width of resection margins influences local recurrence in soft tissue sarcoma patients. Eur J Surg Oncol 2016; 42(6): 899-906.

22. Ecker BL, Peters MG, McMillan MT, Sinnamon AJ, Zhang PJ, Kelz RR et al. Implications of Lymph Node Evaluation in the Management of Resectable Soft Tissue Sarcoma. Ann Surg Oncol 2017; 24(2): 425-433.

23. Sherman KL, Kinnier CV, Farina DA, Wayne JD, Laskin WB, Agulnik M et al. Examination of national lymph node evaluation practices for adult extremity soft tissue sarcoma. J Surg Oncol 2014; 110(6): 682-688.

24. Zigras T, Kupets R, Barbera L, Covens A, Liu Y, Gien LT. Uptake of sentinel lymph node procedures in women with vulvar cancer over time in a population based study. Gynecol Oncol 2019; 153(3): 574-579.

25. Albertsmeier M, Rauch A, Roeder F, Hasenhutl S, Pratschke S, Kirschneck M et al. External Beam Radiation Therapy for Resectable Soft Tissue Sarcoma: A Systematic Review and Meta-Analysis. Ann Surg Oncol 2018; 25(3): 754-767.

26. Le Cesne A, Ouali M, Leahy MG, Santoro A, Hoekstra HJ, Hohenberger P et al. Doxorubicin-based adjuvant chemotherapy in soft tissue sarcoma: pooled analysis of two STBSG-EORTC phase III clinical trials. Ann Oncol 2014; 25(12): 2425-2432.

\section{Table}


Table 1. Clinical-pathological data of the 21 patients with vulvar sarcoma

\begin{tabular}{|c|c|c|c|c|c|c|c|c|c|c|c|}
\hline $\begin{array}{l}\text { Patient } \\
\text { number }\end{array}$ & $\begin{array}{l}\text { Age } \\
\text { (years) }\end{array}$ & $\begin{array}{l}\text { Histological } \\
\text { type }\end{array}$ & $\begin{array}{l}\text { Initial } \\
\text { tumor } \\
\text { Size } \\
\left(\mathrm{cm}^{2}\right)\end{array}$ & $\begin{array}{l}\text { Mitoses } \\
\text { (/10HPF) }\end{array}$ & $\begin{array}{l}\text { Tumor } \\
\text { status } \\
\text { when } \\
\text { referred }\end{array}$ & $\begin{array}{l}\text { Surgical } \\
\text { pattern }\end{array}$ & $\begin{array}{l}\text { Lymphatic } \\
\text { status }\end{array}$ & $\begin{array}{l}\text { Hospital } \\
\text { stay } \\
\text { (days) }\end{array}$ & $\begin{array}{l}\text { Adjuvant } \\
\text { treatment }\end{array}$ & $\begin{array}{l}\text { Follow- } \\
\text { up } \\
\text { (months) }\end{array}$ & $\begin{array}{l}\text { Additional } \\
\text { relapse }\end{array}$ \\
\hline 1 & 24 & LMS & $2 \times 3$ & $<5$ & Resected & WR & N.A. & 7 & $\mathrm{CT}$ & 174 & Yes \\
\hline 2 & 71 & DFSP & $3 \times 4$ & $>20$ & Resected & $W R+I L+F T$ & Positive & 22 & RT & 40 & Yes \\
\hline 3 & 31 & ES & $2 \times 2$ & $>20$ & $\begin{array}{l}\text { Relapse } \\
\text { (first) }\end{array}$ & $W R+I L$ & Negative & 27 & CT & 139 & No \\
\hline 4 & 28 & ES & $1.5 \times 1.5$ & $>20$ & $\begin{array}{l}\text { Relapse } \\
\text { (second) }\end{array}$ & $W R+I L$ & Negative & 25 & CT & 46 & Yes \\
\hline 5 & 62 & ES & $5 \times 6$ & $>20$ & $\begin{array}{l}\text { Relapse } \\
\text { (second) }\end{array}$ & $\mathrm{WR}+\mathrm{FT}$ & N.A. & 23 & CT & 2 & Yes \\
\hline 6 & 54 & DFSP & $1.5 \times 2$ & $<5$ & Resected & $W R+I L$ & Negative & 5 & None & 13 & No \\
\hline 7 & 53 & DFSP & $2 \times 3$ & $<5$ & $\begin{array}{l}\text { Relapse } \\
\text { (third) }\end{array}$ & $\mathrm{WR}+\mathrm{FT}$ & N.A. & 15 & None & 107 & No \\
\hline 8 & 48 & ES & $1 \times 2$ & $>20$ & $\begin{array}{l}\text { Relapse } \\
\text { (third) }\end{array}$ & $W R+I L+R L+F T$ & Positive & 39 & $\mathrm{CT}+\mathrm{RT}$ & 22 & Yes \\
\hline 9 & 46 & AS & $2 \times 3$ & $<5$ & Resected & WR & N.A. & 20 & None & 92 & No \\
\hline 10 & 50 & LMS & $2.5 \times 3$ & $<5$ & Resected & WR+IL & Negative & 25 & None & 88 & No \\
\hline 11 & 21 & DFSP & $3 \times 3.5$ & 5 & Resected & WR & N.A. & 10 & None & 67 & No \\
\hline 12 & 16 & RMS & $4 \times 6$ & $5-10$ & $\begin{array}{l}\text { Relapse } \\
\text { (first) }\end{array}$ & None & N.A. & 2 & $\mathrm{CT}+\mathrm{RT}$ & 30 & Yes \\
\hline 13 & 26 & RMS & $2 \times 3$ & $5-10$ & Resected & WR & N.A. & 6 & None & 60 & No \\
\hline 14 & 45 & LMS & $2 \times 2.5$ & $15-20$ & $\begin{array}{l}\text { Relapse } \\
\text { (first) }\end{array}$ & WR & N.A. & 12 & RT & 56 & No \\
\hline 15 & 22 & ES & $1 \times 1.5$ & $<5$ & Resected & WR & N.A. & 11 & None & 39 & No \\
\hline 16 & 62 & ES & $4 \times 4.5$ & $>20$ & $\begin{array}{l}\text { Relapse } \\
\text { (first) }\end{array}$ & $W R+I L+R L+F T$ & Positive & 22 & $\mathrm{CT}+\mathrm{RT}$ & 6 & Yes \\
\hline 17 & 48 & LMS & $6 \times 6.5$ & $>20$ & Resected & WR & N.A. & 10 & $\mathrm{CT}+\mathrm{RT}$ & 30 & No \\
\hline 18 & 32 & RMS & $5 \times 8$ & $10-15$ & Resident & $W R+I L+F T$ & Negative & 30 & $\mathrm{CT}+\mathrm{RT}$ & 11 & Yes \\
\hline 19 & 43 & ES & $4 \times 4$ & $<5$ & Resected & WR & N.A. & 6 & None & 20 & No \\
\hline 20 & 65 & ES & $3 \times 4$ & $10-15$ & Resected & $W R+I L$ & Negative & 7 & None & 15 & No \\
\hline 21 & 37 & ES & $2 \times 3$ & $5-10$ & Resected & WR & N.A. & 10 & CT & 13 & No \\
\hline
\end{tabular}

Abbreviations: LMS, leiomyosarcoma; DFSP, dermatofibrosarcoma protuberans; ES, epithelioid sarcoma; AS, angiosarcoma, RMS, rhabdomyosarcoma; HPF, $\mathrm{f}$ wide resection; IL, inguinal lymphadenectomy; RL, retroperitoneal lymphadenectomy; FT, flap transplantation; NA, no assessment; CT, chemotherapy; RT, radio evidence of disease; DOD, died of disease; AWD, alive with disease.

\section{Figures}




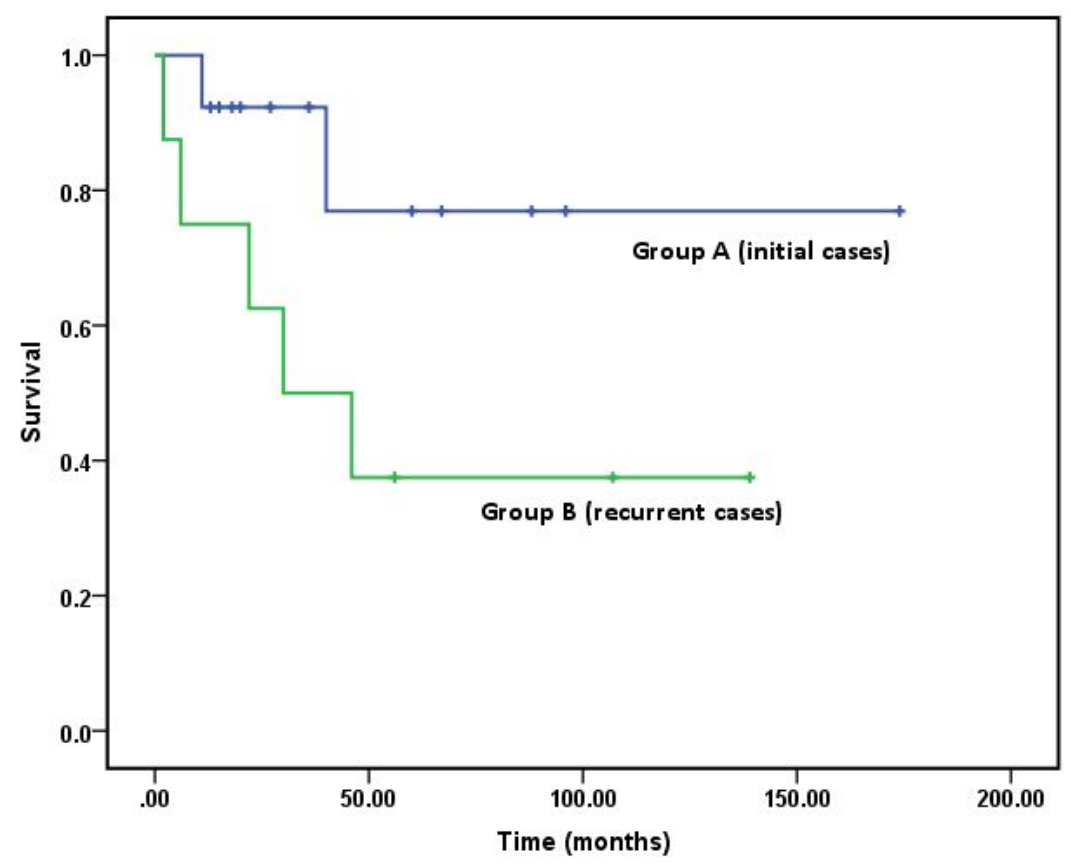

Figure 1

The survival curves for patients referred after initial managements (Group A) and patients referred with recurrence (Group B).

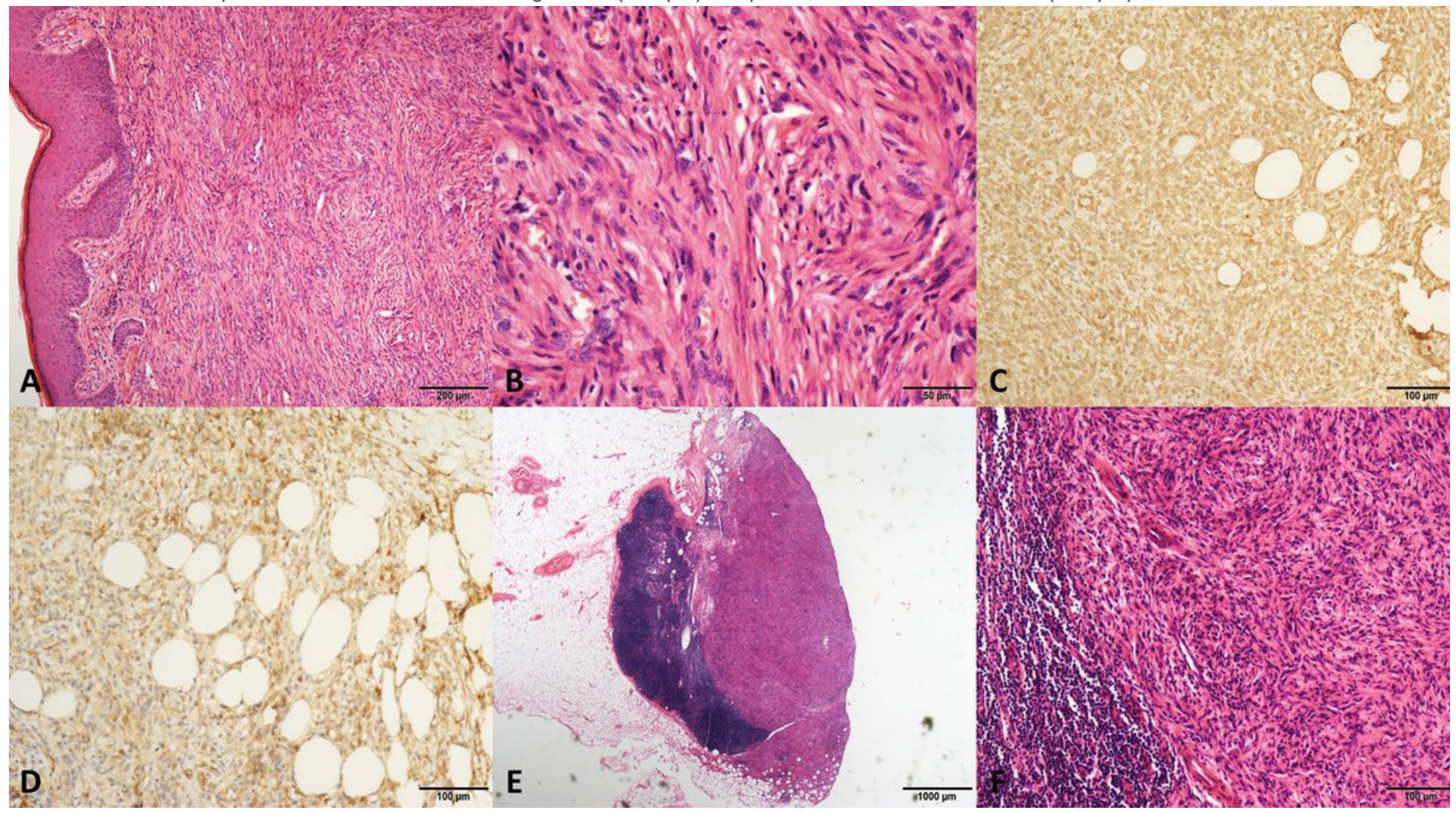

\section{Figure 2}

The pathological graphics of No.2 patient with dermatofibrosaroma protuberans. A. Spindle-shaped cells in a storiform pattern infiltrating the subdermal tissue (hematoxylin/eosin, original magnification $\times 100$ ). B. original magnification $\times 400$. C. positive staining for vimentin (original magnification $\times 200$ ). D. positive staining for CD99 (original magnification $\times 200$ ). E. Metastasis of tumor cells in an inguinal lymph node. (hematoxylin/eosin, original magnification $\times 20)$. F. original magnification $\times 200$.

\section{Supplementary Files}


This is a list of supplementary files associated with this preprint. Click to download.

- Supplementary.docx

Page 9/9 